\title{
A CLASSIFICATION SCHEME FOR NONOSCILLATORY SOLUTIONS OF A HIGHER ORDER NEUTRAL DIFFERENCE EQUATION
}

\author{
ZHI-QIANG ZHU, GEN-QIANG WANG, AND SUI SUN CHENG
}

Received 25 May 2005; Revised 21 September 2005; Accepted 28 September 2005

Nonoscillatory solutions of a nonlinear neutral type higher order difference equations are classified by means of their asymptotic behaviors. By means of the Kranoselskii's fixed point theorem, existence criteria are then provided for justification of such classification.

Copyright (c) 2006 Zhi-qiang Zhu et al. This is an open access article distributed under the Creative Commons Attribution License, which permits unrestricted use, distribution, and reproduction in any medium, provided the original work is properly cited.

\section{Introduction}

Classification schemes for nonoscillatory solutions of nonlinear difference equations are important since further investigations of some of the qualitative behaviors of nonoscillatory solutions can then be reduced to only a number of cases. There are several studies which provide such classification schemes for difference equations, see, for example, [411]. In particular, in [7], a class of nonlinear neutral difference equations of the form

$$
\Delta^{m}\left(x_{n}+c_{n} x_{n-k}\right)+f\left(n, x_{n-l}\right)=0, \quad n=0,1, \ldots,
$$

where $m, k$ and $l$ are integers such that $m \geq 2, k>0$ and $l \geq 0$ is studied and classification schemes are given when $\left\{c_{n}\right\}$ is a nonnegative constant sequence $\left\{c_{0}\right\}$, and in [10], the same equation is studied with odd integer $m \geq 1$, positive integer $k$, integer $l$ and $\left\{c_{n}\right\}=$ $\{-1\}$.

In this paper, we continue our investigation on the possible types of nonoscillatory solutions when $\left\{c_{n}\right\} \subseteq(-1,0]$ and $\lim _{n \rightarrow \infty} c_{n}=c_{0}$ (while the case $\left\{c_{n}\right\} \subseteq(-\infty,-1]$ will be discussed elsewhere). Besides the assumption that $\left\{c_{n}\right\} \subseteq(-1,0]$, we will assume further that $f$ is a continuous function defined on $\{0,1, \ldots\} \times R$ such that $f=f(n, x)$ is nondecreasing in the second variable $x$ and satisfies $x f(n, x)>0$ for $x \neq 0$ and $n \geq 0$.

We will accomplish two things in this paper: to provide a classification scheme for the nonoscillatory solutions of (1.1) in Section 2 and establish in Section 3 sufficient and/or necessary criteria for the existence of solutions in each class. There are no overlapping 
2 A classification scheme for neutral difference equation

results between our paper and [4-11], although some proofs are similar. However, the existence proofs are different in that Cheng-Patula existence theorem is applied in [7], monotone method is used in [10] while we use Krasnoselskii fixed point theorem here. We remark further that classification scheme is also provided for neutral differential equations in [2].

Before we go into details, we will need some preparatory terminologies and results. First of all, given initial $x_{i}$ for $-\max \{k, l\} \leq i \leq 0$, we may calculate from (1.1) $x_{1}, x_{2}, x_{3}, \ldots$ in a recursive manner. Such a sequence $\left\{x_{n}\right\}$ is said to be a solution of (1.1). Among the solutions of (1.1), one is said to be nonoscillatory if it is eventually positive or eventually negative.

Given an integer $a$, it is convenient to set

$$
N(a)=\{a, a+1, a+2, \ldots\} .
$$

Given an integer $\alpha \geq 0$, the generalized factorial function $g(x)=x^{(\alpha)}$ is defined as follows

$$
x^{(\alpha)}= \begin{cases}x(x-1)(x-2) \cdots(x-\alpha+1) & \alpha>0 \\ 1 & \alpha=0\end{cases}
$$

It is well known that $\Delta n^{(\alpha)}=\alpha n^{(\alpha-1)}$ for $\alpha>0$ (see, e.g., [3]).

Let

$$
l_{N_{0}}^{\infty}=\left\{x=\left\{x_{n}\right\}_{n \geq N_{0}}: \sup _{n \geq N_{0}} \frac{\left|x_{n}\right|}{r_{n}}<\infty\right\},
$$

where $N_{0}>0$ is an integer and $\left\{r_{n}\right\}_{n \geq N_{0}}$ is a positive sequence with a uniform positive lower bound. When endowed with the usual linear structure and the norm $\|x\|=$ $\sup _{n \geq N_{0}}\left(\left|x_{n}\right| / r_{n}\right),\left(l_{N_{0}}^{\infty},\|\cdot\|\right)$ is a Banach space. A set $B \subseteq l_{N_{0}}^{\infty}$ is said to be uniformly Cauchy if for any $\varepsilon>0$ there exists an integer $M \geq N_{0}$ such that

$$
\left|\frac{x_{i}}{r_{i}}-\frac{x_{j}}{r_{j}}\right|<\varepsilon \quad i, j>M
$$

for all $x=\left\{x_{n}\right\} \in B$.

Lemma 1.1. A bounded and uniformly Cauchy subset $B \subseteq l_{N_{0}}^{\infty}$ is relatively compact.

Proof. By assumption, we know that for any such $\varepsilon>0$, there exists an integer $M \geq N_{0}>0$ such that for any $x \in B$, we have

$$
\left|\frac{x_{i}}{r_{i}}-\frac{x_{j}}{r_{j}}\right|<\frac{\varepsilon}{3}, \quad i, j \geq M
$$


Let $\Gamma>0$ be a bound for $B$. That is $\|x\| \leq \Gamma$ for all $x \in B$. Choose integers $M_{n}, n=N_{0}, N_{0}+$ $1, \ldots, M$, and numbers $y_{n}^{(1)}<y_{n}^{(2)}<\cdots<y_{n}^{\left(M_{n}\right)}$ such that $y_{n}^{(1)}=-r_{n} \Gamma, y_{n}^{\left(M_{n}\right)}=r_{n} \Gamma$ and

$$
\left|\frac{y_{n}^{(j+1)}}{r_{n}}-\frac{y_{n}^{(j)}}{r_{n}}\right|<\frac{\varepsilon}{3}, \quad 1 \leq j \leq M_{n}-1 .
$$

Now define a sequence $\left\{v_{k}\right\}_{k} \geqslant N_{0}$ as follows. Let $v_{N_{0}}$ be one of the values $\left\{y_{N_{0}}^{(1)}, \ldots, y_{N_{0}}^{\left(M_{N_{0}}\right)}\right\}$, $v_{N_{0}+1}$ be one of the values $\left\{y_{N_{0}+1}^{(1)}, \ldots, y_{N_{0}+1}^{\left(M_{N_{0}+1}\right)}\right\}$. In general, for $N_{0} \leq k \leq M$, let $v_{k}$ equal one of the values $\left\{y_{k}^{(1)}, \ldots, y_{k}^{\left(M_{k}\right)}\right\}$. For $k>M$, let $v_{k}=\left(r_{k} / r_{M}\right) v_{M}$. It is clear that the sequence $\left\{v_{k}\right\}_{k \geqslant N_{0}}$ belongs to $l_{N_{0}}^{\infty}$. Let $L$ be the set of all possible sequences $\left\{v_{k}\right\}_{k \geq N_{0}}$ defined as above. Note that $L$ has $M_{N_{0}} M_{N_{0}+1} \cdots M_{M}$ elements.

We assert that $L$ is a finite $\mathcal{E}$-net for $B$. It is sufficient to show that for any $x=\left\{x_{k}\right\}_{k \geqslant N_{0}} \in$ $B, L$ contains a sequence $v=\left\{v_{k}\right\}_{k \geqslant N_{0}}$ such that

$$
\|x-v\|=\sup _{n \geq N_{0}} \frac{\left|x_{n}-v_{n}\right|}{r_{n}}<\varepsilon .
$$

Indeed, by definition of $L$, we can choose a sequence $\left\{v_{k}\right\}_{k \geqslant N_{0}}$ in $L$ such that

$$
\left|\frac{x_{k}}{r_{k}}-\frac{v_{k}}{r_{k}}\right|<\frac{\varepsilon}{3}, \quad N_{0} \leq k \leq M
$$

Furthermore, by (1.6), (1.9), and the definition of $v=\left\{v_{k}\right\}_{k \geqslant N_{0}}$, for $k>M$, we have

$$
\left|\frac{x_{k}}{r_{k}}-\frac{v_{k}}{r_{k}}\right|=\left|\frac{x_{k}}{r_{k}}-\frac{v_{M}}{r_{M}}\right| \leq\left|\frac{x_{k}}{r_{k}}-\frac{x_{M}}{r_{M}}\right|+\left|\frac{x_{M}}{r_{M}}-\frac{v_{M}}{r_{M}}\right| \leq \frac{\varepsilon}{3}+\frac{\varepsilon}{3}=\frac{2 \varepsilon}{3} .
$$

From (1.9) and (1.10), we see that (1.8) holds. The proof is complete.

Lemma 1.2. Suppose that $\lim _{n \rightarrow \infty} c_{n}=c_{0}$ with $c_{0} \in(-1,0]$ and the sequence $\left\{x_{n} / n^{(i)}\right\}$ is eventually positive or eventually negative, where $i$ is a nonnegative integer. Suppose further that $z_{n}=x_{n}+c_{n} x_{n-k}$ and $\lim _{n \rightarrow \infty}\left(z_{n} / n^{(i)}\right)=b$. Then $\lim _{n \rightarrow \infty}\left(x_{n} / n^{(i)}\right)=b /\left(1+c_{0}\right)$.

Proof. Without loss of generality, we assume that $x_{n} / n^{(i)}>0$ for any positive integer $n$. In case $b$ is finite, we assert that $\left\{x_{n} / n^{(i)}\right\}$ is bounded. Otherwise, there would exist a sequence $\left\{n_{\lambda}\right\}$ of integers with $n_{\lambda} \rightarrow \infty$ for $\lambda \rightarrow \infty$ such that

$$
\lim _{\lambda \rightarrow \infty} \frac{x_{n_{\lambda}}}{n_{\lambda}^{(i)}}=\infty, \quad x_{n} \leq x_{n_{\lambda}}, 0<n \leq n_{\lambda}
$$

On the other hand, we have

$$
\frac{z_{n_{\lambda}}}{n_{\lambda}^{(i)}}=\frac{x_{n_{\lambda}}}{n_{\lambda}^{(i)}}+c_{n_{\lambda}} \frac{x_{n_{\lambda}-k}}{n_{\lambda}^{(i)}} \geq\left(1+c_{n_{\lambda}}\right) \frac{x_{n_{\lambda}}}{n_{\lambda}^{(i)}} \longrightarrow \infty
$$

as $\lambda \rightarrow \infty$. This is contrary to the fact that $b$ is finite. 
4 A classification scheme for neutral difference equation

Let $\lim \sup _{n \rightarrow \infty}\left(x_{n} / n^{(i)}\right)=Q$ and $\liminf _{n \rightarrow \infty}\left(x_{n} / n^{(i)}\right)=q$. Then $0 \leq q \leq Q<\infty$. Moreover, there exist $\left\{n_{\lambda}\right\}$ and $\left\{\bar{n}_{\lambda}\right\}$ such that $\lim _{\lambda \rightarrow \infty} n_{\lambda}=\infty, \lim _{\lambda \rightarrow \infty} \bar{n}_{\lambda}=\infty, \lim _{\lambda \rightarrow \infty}\left(x_{n_{\lambda}} / n_{\lambda}{ }^{(i)}\right)=$ $Q$ and $\lim _{\lambda \rightarrow \infty}\left(x_{\bar{n}_{\lambda}} / \bar{n}_{\lambda}{ }^{(i)}\right)=q$. Note that

$$
\begin{aligned}
b & =\lim _{\lambda \rightarrow \infty} \frac{z_{n_{\lambda}}}{n_{\lambda}{ }^{(i)}}=\lim _{\lambda \rightarrow \infty}\left(\frac{x_{n_{\lambda}}}{n_{\lambda}{ }^{(i)}}+c_{n_{\lambda}} \frac{x_{n_{\lambda}-k}}{n_{\lambda}{ }^{(i)}}\right) \\
& \geq \lim _{\lambda \rightarrow \infty} \frac{x_{n_{\lambda}}}{n_{\lambda}{ }^{(i)}}+\lim _{\lambda \rightarrow \infty} \inf c_{n_{\lambda}} \frac{x_{n_{\lambda}-k}}{\left(n_{\lambda}-k\right)^{(i)}} \frac{\left(n_{\lambda}-k\right)^{(i)}}{n_{\lambda}{ }^{(i)}} \geq Q+c_{0} Q, \\
b & =\lim _{\lambda \rightarrow \infty} \frac{z_{\bar{n}_{\lambda}}}{\bar{n}_{\lambda}^{(i)}}=\lim _{\lambda \rightarrow \infty}\left(\frac{x_{\bar{n}_{\lambda}}}{\bar{n}_{\lambda}{ }^{(i)}}+c_{\bar{n}_{\lambda}} \frac{x_{\bar{n}_{\lambda}-k}}{\bar{n}_{\lambda}{ }^{(i)}}\right) \\
& \leq \lim _{\lambda \rightarrow \infty} \frac{x_{\bar{n}_{\lambda}}}{\bar{n}_{\lambda}{ }^{(i)}}+\lim _{\lambda \rightarrow \infty} \sup c_{\bar{n}_{\lambda}} \frac{x_{\bar{n}_{\lambda}-k}}{\left(\bar{n}_{\lambda}-k\right)^{(i)}} \frac{\left(\bar{n}_{\lambda}-k\right)^{(i)}}{\bar{n}_{\lambda}^{(i)}} \leq q+c_{0} q,
\end{aligned}
$$

we have $\left(1+c_{0}\right) q \geq\left(1+c_{0}\right) Q$. It follows that $q \geq Q$. Hence $q=Q$ and it implies that $\lim _{n \rightarrow \infty}\left(x_{n} / n^{(i)}\right)$ exists. In view of $z_{n}=x_{n}+c_{n} x_{n-k}$ and $\lim _{n \rightarrow \infty}\left(z_{n} / n^{(i)}\right)=b$, we have

$$
\lim _{n \rightarrow \infty} \frac{x_{n}}{n^{(i)}}=\frac{b}{1+c_{0}}
$$

In case $b$ is infinite, then $b=\infty$ or $b=-\infty$. We assert that $b=-\infty$ cannot hold. In fact, for given $c_{1}$ with $-c_{0}<c_{1}<1$, there exists a large integer $N_{0}$ such that $-c_{n} \leq c_{1}$ for $n \geq N_{0}$. Hence, if $b=-\infty$, then $z_{n}=x_{n}+c_{n} x_{n-k}<0$ for $n \geq N$ and

$$
x_{n}<-c_{n} x_{n-k} \leq c_{1} x_{n-k}, \quad n \geq N,
$$

where $N \geq N_{0}$ is some positive integer. It implies that

$$
0<x_{N+\lambda k}<c_{1} x_{N+(\lambda-1) k}<\cdots<c_{1}^{\lambda} x_{N} .
$$

So that $\lim _{\lambda \rightarrow \infty} x_{N+\lambda k}=0$. Thus

$$
\lim _{\lambda \rightarrow \infty} z_{N+\lambda k}=0
$$

which implies that $b=-\infty$ is impossible.

Now, for arbitrary $M>0$, there exists a sufficiently large integer $N$ such that

$$
\frac{z_{n}}{n^{(i)}}=\frac{x_{n}}{n^{(i)}}+c_{n} \frac{x_{n-k}}{n^{(i)}} \geq M, \quad n \geq N .
$$

It follows that

$$
\frac{x_{n}}{n^{(i)}} \geq M, \quad n \geq N
$$

That is $\lim _{n \rightarrow \infty}\left(x_{n} / n^{(i)}\right)=\infty$. The proof is complete.

The following two propositions are respectively in [1, Theorems 1.7.9 and 1.7.11]. 
Lemma 1.3. Suppose that the sequence $\left\{x_{n}\right\}$ and $\left\{y_{n}\right\}$ satisfy the following conditions,

(i) $y_{n}>0$ and $\Delta y_{n}>0$ for all large integers $n$ and $\lim _{n \rightarrow \infty} y_{n}=\infty$, and

(ii) $\lim _{n \rightarrow \infty}\left(\Delta x_{n} / \Delta y_{n}\right)=b$.

Then $\lim _{n \rightarrow \infty}\left(x_{n} / y_{n}\right)=\lim _{n \rightarrow \infty}\left(\Delta x_{n} / \Delta y_{n}\right)=b$, where $b$ can be finite or infinite.

Lemma 1.4. Let $u=u(n)$ be a sequence defined for $n \in N(a), u(n)>0$ with $\Delta^{m} u(n)$ of constant sign on $N(a)$ and not identically zero. Then, there exists an integer $m^{*}, 0 \leq m^{*} \leq m$ with $m+m^{*}$ odd for $\Delta^{m} u(n) \leq 0$ or, $m+m^{*}$ even for $\Delta^{m} u(n) \geq 0$ and such that

$$
\begin{gathered}
m^{*} \leq m-1 \text { implies }(-1)^{m^{*}+i} \Delta^{i} u(n)>0 \quad \forall n \in N(a), m^{*}+1 \leq i \leq m, \\
m^{*} \geq 1 \text { implies } \Delta^{i} u(n)>0 \quad \forall \text { large } n \in N(a), 1 \leq i \leq m^{*} .
\end{gathered}
$$

Remark 1.5. If $u(n)<0$ in Lemma 1.4 , then there exists $m^{*}, 0 \leq m^{*} \leq m$ with $m+m^{*}$ odd for $\Delta^{m} u(n) \geq 0$ or, $m+m^{*}$ even for $\Delta^{m} u(n) \leq 0$ and such that

$$
\begin{gathered}
m^{*} \leq m-1 \text { implies }(-1)^{m^{*}+i} \Delta^{i} u(n)<0 \quad \forall n \in N(a), m^{*}+1 \leq i \leq m, \\
m^{*} \geq 1 \text { implies } \Delta^{i} u(n)<0 \quad \forall \text { large } n \in N(a), 1 \leq i \leq m^{*} .
\end{gathered}
$$

Lemma 1.6 (Kranoselskii's fixed point theorem). Suppose $B$ is a Banach space and $\Omega$ is a bounded, convex and closed subset of $B$. Let $U, S: \Omega \rightarrow B$ satisfy the following conditions.

(i) $U x+S y \in \Omega$ for any $x, y \in \Omega$,

(ii) $U$ is a contraction mapping, and

(iii) $S$ is completely continuous.

Then $U+S$ has a fixed point in $\Omega$.

\section{Classifications of nonoscillatory solutions}

In the following discussions, we assume throughout that

$$
\lim _{n \rightarrow \infty} c_{n}=c_{0} \in(-1,0] .
$$

We set

$$
z_{n}=x_{n}+c_{n} x_{n-k}
$$

whenever it is defined. Equation (1.1) can now be written as

$$
\Delta^{m} z_{n}=-f\left(n, x_{n-l}\right) .
$$

We will propose a classification scheme for the nonoscillatory solutions of (1.1). For this purpose, we first note that if $x=\left\{x_{n}\right\}$ is an eventually negative solution of (1.1), then $y=\left\{y_{n}\right\}$ defined by $y_{n}=-x_{n}$ will satisfy

$$
\Delta^{m}\left(y_{n}+c y_{n-k}\right)+\tilde{f}\left(n, y_{n-l}\right)=0,
$$

where

$$
\tilde{f}(n, u)=-f(n,-u), \quad n \in N(0), u \in R
$$


has the same properties satisfied by $f$, that is, $\tilde{f}$ is a continuous function defined on $\{0,1, \ldots\} \times R$ such that $\tilde{f}=\tilde{f}(n, u)$ is nondecreasing in the second variable $u$ and satisfies $u \tilde{f}(n, u)>0$ for $u \neq 0$ and $n \geq 0$. Therefore, we may restrict our attention to the set $S^{+}$of all eventually positive solutions of (1.1). Motivated by the classification scheme in [2], we make use of the following notations for classifying our eventually positive solutions:

$$
\begin{gathered}
A_{k}(\alpha, \beta)=\left\{\left\{x_{n}\right\} \in S^{+}: \lim _{n \rightarrow \infty} \frac{x_{n}}{n^{(k-1)}}=\alpha, \lim _{n \rightarrow \infty} \frac{x_{n}}{n^{(k)}}=\beta\right\}, \quad k \geq 1, \\
A_{0}(\alpha)=\left\{\left\{x_{n}\right\} \in S^{+}: \lim _{n \rightarrow \infty} x_{n}=\alpha\right\} .
\end{gathered}
$$

Theorem 2.1. (a) Suppose that $m$ is even. If $x=\left\{x_{n}\right\}$ is an eventually positive solution of (1.1), then either $x \in A_{0}(0)$ or there are some $j \in\{1,2, \ldots, m / 2\}$ and $a>0$ such that $x$ belongs to $A_{2 j-1}(\infty, a), A_{2 j-1}(\infty, 0)$ or $A_{2 j-1}(a, 0)$. (b) Suppose that $m$ is odd. If $x=\left\{x_{n}\right\}$ is an eventually positive solution of (1.1), then either $x$ belongs to $A_{0}(\alpha)$ for some $\alpha \geq 0$, or there are $j \in\{1,2, \ldots,(m-1) / 2\}$ and $a>0$ such that $x$ belongs to $A_{2 j}(\infty, a), A_{2 j}(\infty, 0)$ or $A_{2 j}(a, 0)$.

Proof. Let $m$ is even and $x=\left\{x_{n}\right\}$ be an eventually positive solution of (1.1). Then, in view of (2.3), there exists some integer $N>0$ such that $\Delta^{m} z_{n}<0$ for $n \geq N$. Therefore, $z_{n}$ is eventually of fixed sign. For the sake of simplicity, we may assume that $\left\{z_{n}\right\}$ is of fixed sign for $n \geq N$.

First of all, suppose $z_{n}<0$ for $n \geq N$. By the same reasoning as in the proof of Lemma 1.2 , we may show that

$$
\lim _{\lambda \rightarrow \infty} z_{N+\lambda k}=0
$$

On the other hand, in view of Lemma 1.4, there exists some even $m^{*}$ with $0 \leq m^{*} \leq m$ such that eventually $\Delta^{i} z_{n}<0$ for $0 \leq i \leq m^{*}$ and $(-1)^{m^{*}+i} \Delta^{i} z_{n}<0$ for $m^{*}+1 \leq i \leq m$. There are now two cases to consider.

Case $1\left(m^{*}=0\right)$. Then we have eventually

$$
z_{n}<0, \quad \Delta z_{n}>0
$$

By (2.8), we can set

$$
\lim _{n \rightarrow \infty} z_{n}=L_{0} \leq 0
$$

In view of (2.7), we find that $\lim _{n \rightarrow \infty} z_{n}=0$. By Lemma 1.2, we have $\lim _{n \rightarrow \infty} x_{n}=0$. Hence $x$ belongs to $A_{0}(0)$.

Case $2\left(m^{*} \geq 2\right)$. Then we have eventually

$$
z_{n}<0, \quad \Delta z_{n}<0
$$

It implies $\lim _{n \rightarrow+\infty} z_{n}<0$ which is contrary to (2.7). Hence $m^{*} \geq 2$ does not hold. 
Now we suppose $z_{n}>0$ for $n \geq N$. Similar to the proof in [7, Theorem 1], we may see that $x$ belongs to $A_{2 j-1}(\infty, a), A_{2 j-1}(\infty, 0)$ or $A_{2 j-1}(a, 0)$ for some $j \in\{1,2, \ldots, m / 2\}$ and $a>0$.

When $m$ is odd, the proof is similar to those above and hence is skipped. The proof is complete.

\section{Existence criteria}

Eventually positive (and by analog eventually negative) solutions of (1.1) have been classified according to Theorem 2.1. We now justify our classification schemes by finding existence criteria for each type of solutions.

Theorem 3.1. Suppose that $m$ is even. If (1.1) has a solution in $A_{2 j-1}(\infty, a)$ for some $j \in$ $\{1,2, \ldots, m / 2\}$ and $a>0$, then there exists some $K>0$ such that

$$
\sum_{i=0}^{\infty} \frac{(i+m-2 j)^{(m-2 j)}}{(m-2 j) !} f\left(i, K(i-l)^{(2 j-1)}\right)<\infty .
$$

The converse is also true.

Proof. First of all, we remark that

$$
\sum_{i_{\lambda}=n}^{\infty} \sum_{i_{\lambda-1}=i_{\lambda}}^{\infty} \cdots \sum_{i_{2}=i_{3}}^{\infty} \sum_{i_{1}=i_{2}}^{\infty} f\left(i_{1}, x_{i_{1}-l}\right)=\sum_{i=n}^{\infty} \frac{(i-n+\lambda-1)^{(\lambda-1)}}{(\lambda-1) !} f\left(i, x_{i-l}\right) .
$$

Let $x=\left\{x_{n}\right\}$ be an eventually positive solution of (1.1) in $A_{2 j-1}(\infty, a)$. Then we may suppose that there exists an integer $N_{0}>0$ such that $x_{n}>0$ and $x_{n-l}>0$ for $n>N_{0}$. In view of (2.3), we have $\Delta^{m} z_{n}<0$ for $n>N_{0}$. Thereby $\left\{\Delta^{i} z_{n}\right\}$ is eventually monotonic for $i=0,1,2, \ldots, m-1$. Since $\lim _{n \rightarrow \infty}\left(x_{n} / n^{(2 j-1)}\right)=a>0$, there exists some integer $N_{1}>N_{0}$ such that

$$
\frac{1}{2} a n^{(2 j-1)} \leq x_{n} \leq \frac{3}{2} a n^{(2 j-1)}, \quad n \geq N_{1} .
$$

Note that $\lim _{n \rightarrow \infty}\left(z_{n} / n^{(2 j-1)}\right)=\left(1+c_{0}\right) a$ implies

$$
\lim _{n \rightarrow \infty} \Delta^{2 j-1} z_{n}=\left(1+c_{0}\right) a(2 j-1) !
$$

By (3.4) and the monotonicity of $\Delta^{i} z_{n}$, we have

$$
\lim _{n \rightarrow \infty} \Delta^{i} z_{n}=0, \quad i=2 j, 2 j+1, \ldots, m-1 .
$$

Summing (2.3) $m-2 j$ times from $n$ to $N_{1}$ and invoking (3.5) in each time, we obtain

$$
\begin{aligned}
\Delta^{2 j} z_{n} & =-\sum_{i_{m-2 j}=n}^{\infty} \cdots \sum_{i_{2}=i_{3}}^{\infty} \sum_{i_{1}=i_{2}}^{\infty} f\left(i_{1}, x_{i_{1}-l}\right) \\
& =-\sum_{i=n}^{\infty} \frac{(i-n+m-2 j-1)^{(m-2 j-1)}}{(m-2 j-1) !} f\left(i, x_{i-l}\right), \quad n \geq N_{1} .
\end{aligned}
$$


8 A classification scheme for neutral difference equation

Summing the above equation again from $N_{1}$ to $n$, we obtain

$$
\Delta^{2 j-1} z_{n+1}=\Delta^{2 j-1} z_{N_{1}}-\sum_{i_{2}=N_{1}}^{n} \sum_{i_{1}=i_{2}}^{\infty} \frac{\left(i_{1}-i_{2}+m-2 j-1\right)^{(m-2 j-1)}}{(m-2 j-1) !} f\left(i_{1}, x_{i_{1}-l}\right) .
$$

By (3.4), the above equation implies that

$$
\sum_{i_{2}=n}^{\infty} \sum_{i_{1}=i_{2}}^{\infty} \frac{\left(i_{1}-i_{2}+m-2 j-1\right)^{(m-2 j-1)}}{(m-2 j-1) !} f\left(i_{1}, x_{i_{1}-l}\right)<\infty, \quad n \geq N_{1} .
$$

That is,

$$
\sum_{i=n}^{\infty} \frac{(i-n+m-2 j)^{(m-2 j)}}{(m-2 j) !} f\left(i, x_{i-l}\right)<\infty, \quad n \geq N_{1} .
$$

Let $K=a / 2$. In view of (3.3), (3.9) and the monotonicity of $f(n, x)$ in $x$, we see that (3.1) holds.

Conversely, suppose (3.1) holds for some $K>0$. Set $R_{n}=n^{(2 j-1)}$. In view of (3.2), we have

$$
\begin{array}{r}
\sum_{i_{m-2 j+1}=n}^{\infty} \sum_{i_{m-2 j}=i_{m-2 j+1}}^{\infty} \cdots \sum_{i_{2}=i_{3}}^{\infty} \sum_{i_{1}=i_{2}}^{\infty} f\left(i_{1}, K\left(i_{1}-l\right)^{(2 j-1)}\right) \\
=\sum_{i=n}^{\infty} \frac{(i-n+m-2 j)^{(m-2 j)}}{(m-2 j) !} f\left(i, K(i-l)^{(2 j-1)}\right) .
\end{array}
$$

Note that (2.1), there are two cases to consider.

In case $-1<c_{0}<0$, take $c_{1}$ so that $-c_{0}<c_{1}<\left(1-4 c_{0}\right) / 5<1$. Then $\left(1-5 c_{1}\right) /\left(4 c_{0}\right)<$ 1. Note that $\lim _{n \rightarrow \infty}\left(\left|c_{n}\right| R_{n} / R_{n-k-l}\right)=\left|c_{0}\right|, \lim _{n \rightarrow \infty}\left(R_{n-k} / R_{n}\right)=1$ and (3.1) holds. Thus there exists an integer $N>k+l$ such that when $n \geq N$, we have

$$
\begin{aligned}
& \frac{\left|c_{n}\right| R_{n}}{R_{n-k-l}} \leq c_{1}, \\
& -c_{n} \leq c_{1}, \\
& \frac{R_{n-k}}{R_{n}} \geq \frac{1-5 c_{1}}{4 c_{n}}, \\
& \sum_{i_{m-2 j+1}=N}^{\infty} \sum_{i_{m-2 j}=i_{m-2 j+1}}^{\infty} \cdots \sum_{i_{1}=i_{2}}^{\infty} f\left(i_{1}, K\left(i_{1}-l\right)^{(2 j-1)}\right)<\frac{\left(1-c_{1}\right) K}{8} \text {. }
\end{aligned}
$$

Take $N_{0}=N-k-l, r_{n}=R_{n}^{2}$ and define the Banach space $l_{N_{0}}^{\infty}$ as in (1.4). Let

$$
\Omega=\left\{x \in l_{N_{0}}^{\infty}: \frac{1}{2} K R_{n} \leq x_{n} \leq K R_{n}\right\}
$$


Then it is obvious that $\Omega$ is a bounded, convex and closed subset of $l_{N_{0}}^{\infty}$, and for any $x \in$ $\Omega$ and $n \geq N_{0}+l$, we have

$$
f\left(n, x_{n-l}\right) \leq f\left(n, K(n-l)^{(2 j-1)}\right) .
$$

Define operators $U$ and $S$ on $\Omega$ as follows:

$$
\begin{aligned}
& (U x)_{n}= \begin{cases}-\frac{3}{4} c_{1} K R_{n}-\frac{c_{N} x_{N-k}}{R_{N}} R_{n} & N_{0} \leq n<N \\
-\frac{3}{4} c_{1} K R_{n}-c_{n} x_{n-k} & n \geq N,\end{cases} \\
& (S x)_{n}= \begin{cases}\frac{3}{4} K R_{n} & N_{0} \leq n<N \\
\frac{3}{4} K R_{n}+F(n) & n \geq N,\end{cases}
\end{aligned}
$$

where

$$
F(n)=\sum_{i_{m}=N}^{n-1} \cdots \sum_{i_{m-2 j+3}=N}^{i_{m-2 j+4}-1} \sum_{i_{m-2 j+2}=N}^{i_{m-2 j+3}-1} \sum_{i_{m-2 j+1}=i_{m-2 j+2}}^{\infty} \sum_{i_{m-2 j}=i_{m-2 j+1}}^{\infty} \cdots \sum_{i_{1}=i_{2}}^{\infty} f\left(i_{1}, x_{i_{1}-l}\right) .
$$

In view of (3.16) and (3.14),we have

$$
F(n) \leq \sum_{i_{m}=N}^{n-1} \cdots \sum_{i_{m-2 j+3}=N}^{i_{m-2 j+4}-1} \sum_{i_{m-2 j+2}=N}^{i_{m-2 j+3}-1} \frac{\left(1-c_{1}\right) K}{8}=\frac{\left(1-c_{1}\right) K(n-N)^{(2 j-1)}}{8(2 j-1) !} \leq \frac{\left(1-c_{1}\right) K}{8} R_{n}
$$

for $n \geq N$.

Next, we will show that the operators $U$ and $S$ satisfy the conditions of Kranoselskii's fixed point theorem.

First, we claim that $U x+S y \in \Omega$ for any $x, y \in \Omega$. Indeed, for $N_{0} \leq n<N$, in view of (3.13) and (3.12), we have

$$
\begin{aligned}
& (U x)_{n}+(S y)_{n}=\left(\frac{3}{4}\left(1-c_{1}\right) K-c_{N} \frac{x_{N-k}}{R_{N}}\right) R_{n} \geq\left(\frac{3}{4}\left(1-c_{1}\right) K-c_{N} K \frac{R_{N-k}}{2 R_{N}}\right) R_{n} \geq \frac{1}{2} K R_{n}, \\
& (U x)_{n}+(S y)_{n} \leq\left(\frac{3}{4}\left(1-c_{1}\right) K-c_{N} K \frac{R_{N-k}}{R_{N}}\right) R_{n} \leq\left(\frac{3}{4}\left(1-c_{1}\right)+c_{1}\right) K R_{n} \leq K R_{n} .
\end{aligned}
$$

When $n \geq N$, invoking (3.13) again, we have

$$
(U x)_{n}+(S y)_{n} \geq \frac{3}{4}\left(1-c_{1}\right) K R_{n}-c_{n} x_{n-k} \geq \frac{3}{4}\left(1-c_{1}\right) K R_{n}-c_{n} \frac{1}{2} K \frac{R_{n-k}}{R_{n}} R_{n} \geq \frac{1}{2} K R_{n}
$$


10 A classification scheme for neutral difference equation

and, in view of (3.19) and (3.12), we have

$$
\begin{aligned}
(U x)_{n}+(S y)_{n} & \leq \frac{3}{4}\left(1-c_{1}\right) K R_{n}-c_{n} x_{n-k}+\frac{\left(1-c_{1}\right) K}{8} R_{n} \\
& \leq \frac{3}{4}\left(1-c_{1}\right) K R_{n}-c_{n} K R_{n-k}+\frac{\left(1-c_{1}\right) K}{8} R_{n} \leq K R_{n}
\end{aligned}
$$

That is, $U x+S y \in \Omega$ for any $x, y \in \Omega$.

Let $x, y \in \Omega$. In view of (3.11), we have

$$
\begin{aligned}
\frac{1}{R_{n}^{2}}\left|(U x)_{n}-(U y)_{n}\right| & =\frac{\left|c_{N}\right|\left|x_{N-k}-y_{N-k}\right|}{R_{N} R_{n}} \\
& =\frac{\left|x_{N-k}-y_{N-k}\right|}{R_{N-k}^{2}} \frac{\left|c_{N}\right| R_{N-k}^{2}}{R_{N} R_{n}} \leq c_{1} \sup _{n \geq N_{0}} \frac{\left|x_{n}-y_{n}\right|}{R_{n}^{2}}
\end{aligned}
$$

for $N_{0} \leq n<N$. And, for $n \geq N$, we have

$$
\frac{1}{R_{n}^{2}}\left|(U x)_{n}-(U y)_{n}\right| \leq\left|c_{n}\right| \sup _{n \geq N_{0}} \frac{\left|x_{n}-y_{n}\right|}{R_{n}^{2}} .
$$

Therefore, we have

$$
\|U x-U y\| \leq c_{1}\|x-y\|
$$

for any $x, y \in \Omega$. Hence, $U$ is a contraction mapping.

Next, we will prove that $S$ is a completely continuous mapping. Indeed, it is obvious that $(S x)_{n} \geq(K / 2) R_{n}$ for $n \geq N_{0}$ and $(S x)_{n} \leq K R_{n}$ for $N_{0} \leq n<N$. When $n \geq N$, by means of (3.19), we have

$$
(S x)_{n} \leq \frac{3}{4} K R_{n}+\frac{\left(1-c_{1}\right) K}{8} R_{n} \leq K R_{n}
$$

That is, the operator $S$ maps $\Omega$ into $\Omega$.

Now we consider the continuity of $S$. Let $x^{(\lambda)} \in \Omega$ and $\left\|x^{(\lambda)}-x\right\| \rightarrow 0$ when $\lambda \rightarrow \infty$, we assert that $S x^{(\lambda)}$ converges to $S x$ by $\|\cdot\|$. Indeed, $\left\|x^{(\lambda)}-x\right\| \rightarrow 0$ implies that $x \in \Omega$ and $\left|x_{n}^{(\lambda)}-x_{n}\right| \rightarrow 0$ when $\lambda \rightarrow \infty$ for any integer $n \geq N_{0}$. Thereby, we have

$$
\left|f\left(n, x_{n-l}^{(\lambda)}\right)-f\left(n, x_{n-l}\right)\right| \longrightarrow 0, \quad \lambda \longrightarrow \infty
$$

for any integer $n \geq N_{0}+l$. By definition of $S$, we have

$$
\left|\left(S x^{(\lambda)}\right)_{n}-(S x)_{n}\right|=0
$$

for $N_{0} \leq n<N$ and

$$
\left|\left(S x^{(\lambda)}\right)_{n}-(S x)_{n}\right| \leq H_{\lambda}(n)
$$


for $n \geq N$, where

$$
H_{\lambda}(n)=\sum_{i_{m}=N}^{n-1} \cdots \sum_{i_{m-2 j+3}=N}^{i_{m-2 j+4}-1} \sum_{i_{m-2 j+2}=N}^{i_{m-2 j+3}-1} \sum_{i_{m-2 j+1}=i_{m-2 j+2}}^{\infty} \sum_{i_{m-2 j}=i_{m-2 j+1}}^{\infty} \cdots \sum_{i_{1}=i_{2}}^{\infty}\left|f\left(i_{1}, x_{i_{1}-l}^{(\lambda)}\right)-f\left(i_{1}, x_{i_{1}-l}\right)\right| .
$$

In view of (3.16), we have

$$
\left|f\left(i_{1}, x_{i_{1}-l}^{(\lambda)}\right)-f\left(i_{1}, x_{i_{1}-l}\right)\right| \leq 2 f\left(i_{1}, K\left(i_{1}-l\right)^{(2 j-1)}\right), \quad n \geq N_{0}+l .
$$

Thus

$$
\begin{aligned}
H_{\lambda}(n) & \leq \sum_{i_{m}=N}^{n-1} \cdots \sum_{i_{m-2 j+3}=N}^{i_{m-2 j+4}-1} \sum_{i_{m-2 j+2}=N}^{i_{m-2 j+3}-1} \sum_{i_{m-2 j+1}=N}^{\infty} \sum_{i_{m-2 j}=i_{m-2 j+1}}^{\infty} \cdots \sum_{i_{1}=i_{2}}^{\infty}\left|f\left(i_{1}, x_{i_{1}-l}^{(\lambda)}\right)-f\left(i_{1}, x_{i_{1}-l}\right)\right| \\
& \leq R_{n} \sum_{i_{m-2 j+1}=N}^{\infty} \sum_{i_{m-2 j}=i_{m-2 j+1}}^{\infty} \cdots \sum_{i_{1}=i_{2}}^{\infty}\left|f\left(i_{1}, x_{i_{1}-l}^{(\lambda)}\right)-f\left(i_{1}, x_{i_{1}-l}\right)\right| .
\end{aligned}
$$

To sum up, we have

$$
\begin{aligned}
\left\|\left(S x^{(\lambda)}\right)_{n}-(S x)_{n}\right\| & \leq \sup _{n \geq N_{0}} \frac{1}{R_{n}} \sum_{i_{m-2 j+1}=N}^{\infty} \sum_{i_{m-2 j}=i_{m-2 j+1}}^{\infty} \cdots \sum_{i_{1}=i_{2}}^{\infty}\left|f\left(i_{1}, x_{i_{1}-l}^{(\lambda)}\right)-f\left(i_{1}, x_{i_{1}-l}\right)\right| \\
& =\sup _{n \geq N_{0}} \frac{1}{R_{n}} \sum_{i=N}^{\infty} \frac{(i-N+m-2 j)^{(m-2 j)}}{(m-2 j)}\left|f\left(i, x_{i-l}^{(\lambda)}\right)-f\left(i, x_{i-l}\right)\right| .
\end{aligned}
$$

In view of (3.27) and (3.31), the Lebesque's dominated theorem [3] then implies $\lim _{\lambda \rightarrow \infty}\left\|\left(S x^{\lambda}\right)-(S x)\right\|=0$. This means $S$ is continuous.

Finally, we prove that $S \Omega$ is relatively compact. We assert that $S \Omega$ is uniformly Cauchy. Indeed, for any $\varepsilon>0$, there exists $N_{1}>N$ such that $1 / R_{n}<\varepsilon / 3 K$ for $n \geq N_{1}$. For any $x \in \Omega$ and $i_{1}, i_{2} \geq N_{1}$, in view of (3.19), we have that

$$
\left|\frac{(S x)_{i_{1}}}{R_{i_{1}}^{2}}-\frac{(S x)_{i_{2}}}{R_{i_{2}}^{2}}\right| \leq \frac{(S x)_{i_{1}}}{R_{i_{1}}^{2}}+\frac{(S x)_{i_{2}}}{R_{i_{2}}^{2}} \leq \frac{3 K}{4}\left(R_{i_{1}}^{-1}+R_{i_{2}}^{-1}\right)+\sum_{j=1}^{2} \frac{\left(1-c_{1}\right) K}{8 R_{i_{j}}} \leq \frac{\varepsilon}{2}+\frac{\varepsilon}{12}<\varepsilon .
$$

By Lemma 1.1, $S \Omega$ is relatively compact.

To sum up, we have proved that $S$ is a completely continuous mapping. 
12 A classification scheme for neutral difference equation

By the Kranoselskii's fixed point theorem, there then exists $x=\left\{x_{n}\right\} \in \Omega$ such that $(U x)_{n}+(S x)_{n}=x_{n}$. Therefore, we have

$$
x_{n}=\frac{3}{4}\left(1-c_{1}\right) K R_{n}-c_{n} x_{n-k}+F(n), \quad n \geq N .
$$

It is easy to verify that $x_{n}$ satisfy (1.1). Furthermore, we have

$$
\begin{aligned}
\Delta^{2 j-1} F(n) & =\sum_{i_{m-2 j+1}=n}^{\infty} \sum_{i_{m-2 j}=i_{m-2 j+1}}^{\infty} \cdots \sum_{i_{1}=i_{2}}^{\infty} f\left(i_{1}, x_{i_{1}-l}\right) \\
& \leq \sum_{i_{m-2 j+1}=n}^{\infty} \sum_{i_{m-2 j}=i_{m-2 j+1}}^{\infty} \cdots \sum_{i_{1}=i_{2}}^{\infty} f\left(i_{1}, K\left(i_{1}-l\right)^{(2 j-1)}\right) .
\end{aligned}
$$

In view of (3.1) and (3.10), we have

$$
\lim _{n \rightarrow \infty} \Delta^{2 j-1} F(n)=0,
$$

so that

$$
\lim _{n \rightarrow \infty} \frac{F(n)}{n^{(2 j-1)}}=0
$$

Now we turn to (3.35) and obtain

$$
\lim _{n \rightarrow \infty} \frac{z_{n}}{n^{(2 j-1)}}=\frac{3}{4}\left(1-c_{1}\right) K
$$

By Lemma 1.2, we have

$$
\lim _{n \rightarrow \infty} \frac{x_{n}}{n^{(2 j-1)}}=\frac{3\left(1-c_{1}\right) K}{4\left(1+c_{0}\right)},
$$

which infers that $\lim _{n \rightarrow \infty}\left(x_{n} / n^{(2 j-2)}\right)=\infty$. In summary, (1.1) has a solution in $A_{2 j-1}(\infty, a)$ when $-1<c_{0}<0$.

In case $c_{0}=0$, take $c_{1}$ so that $0<c_{1} \leq 1 / 3$. Then, there exists an integer $N>k+l$ such that when $n \geq N$, (3.11) to (3.14) hold. Take operators $U$ and $S$ to be the same operators as above. Then we may prove in similar manners that (1.1) has a solution in $A_{2 j-1}(\infty, a)$. The proof is complete.

A similar theorem holds when $m$ is odd, the proof is similar to that of Theorem 3.1 and hence is skipped.

Theorem 3.2. Suppose $m$ is odd. If (1.1) has a solution in $A_{2 j}(\infty, a)$ for some $j \in\{1,2, \ldots$, $(m-1) / 2\}$ and $a>0$, then there exists some $K>0$ such that

$$
\sum_{i=0}^{\infty} \frac{(i+m-2 j-1)^{(m-2 j-1)}}{(m-2 j-1) !} f\left(i, K(i-l)^{(2 j)}\right)<\infty .
$$

The converse also holds. 
Theorem 3.3. Suppose that $m$ is even. If (1.1) has an eventually positive solution in $A_{2 j-1}(a$, 0) for some $j \in\{1, \ldots, m / 2\}$ and $a>0$, then there is some $K>0$ such that

$$
\sum_{i=0}^{\infty} \frac{(i+m-2 j+1)^{(m-2 j+1)}}{(m-2 j+1) !} f\left(i, K(i-l)^{(2 j-2)}\right)<\infty .
$$

The converse is also true.

The proof is similar to that of Theorem 3.1 by taking $R_{n}=n^{(2 j-2)}$.

Theorem 3.4. Suppose $m$ is odd. If (1.1) has a solution in $A_{2 j}(a, 0)$ for some $j \in\{1,2, \ldots$, $(m-1) / 2\}$ and $a>0$, then there is some $K>0$ such that

$$
\sum_{i=0}^{\infty} \frac{(i+m-2 j)^{(m-2 j)}}{(m-2 j) !} f\left(i, K(i-l)^{(2 j-1)}\right)<\infty .
$$

The converse also holds.

Theorem 3.5. Suppose that $m$ is even. If (1.1) has a solution in $A_{2 j-1}(\infty, 0)$ for some $j \in$ $\{1,2, \ldots, m / 2\}$, then

$$
\begin{gathered}
\sum_{i=0}^{\infty} \frac{(i+m-2 j)^{(m-2 j)}}{(m-2 j) !} f\left(i,(i-l)^{(2 j-2)}\right)<\infty, \\
\sum_{i=0}^{\infty} \frac{(i+m-2 j+1)^{(m-2 j+1)}}{(m-2 j+1) !} f\left(i,(i-l)^{(2 j-1)}\right)=\infty .
\end{gathered}
$$

Conversely, if there is some $j \in\{1,2, \ldots, m / 2\}$ such that

$$
\begin{gathered}
\sum_{i=0}^{\infty} \frac{(i+m-2 j)^{(m-2 j)}}{(m-2 j) !} f\left(i,(i-l)^{(2 j-1)}\right)<\infty, \\
\sum_{i=0}^{\infty} \frac{(i+m-2 j+1)^{(m-2 j+1)}}{(m-2 j+1) !} f\left(i,(i-l)^{(2 j-2)}\right)=\infty,
\end{gathered}
$$

then (1.1) has a solution in $A_{2 j-1}(\infty, 0)$.

Proof. Let $x=\left\{x_{n}\right\}$ be an eventually positive solution of (1.1) in $A_{2 j-1}(\infty, 0)$. Note that $\lim _{n \rightarrow \infty}\left(x_{n} / n^{(2 j-1)}\right)=0, \lim _{n \rightarrow \infty}\left(x_{n} / n^{(2 j-2)}\right)=\infty$ and (2.3) holds. Therefore there exists an integer $N_{0}>0$ such that

$$
\begin{array}{cc}
\Delta^{m} z_{n}<0, & n \geq N_{0}, \\
x_{n} \leq n^{(2 j-1)}, & n \geq N_{0}, \\
x_{n} \geq n^{(2 j-2)}, & n \geq N_{0} .
\end{array}
$$


14 A classification scheme for neutral difference equation

In view of (3.48), $\left\{\Delta^{i} z_{n}\right\}$ is eventually monotonic for $i=0,1,2, \ldots, m-1$. Since $\lim _{n \rightarrow \infty}\left(z_{n} /\right.$ $\left.n^{(2 j-1)}\right)=0$ and $\lim _{n \rightarrow \infty}\left(z_{n} / n^{(2 j-2)}\right)=\infty$, we have

$$
\begin{aligned}
& \lim _{n \rightarrow \infty} \Delta^{2 j-1} z_{n}=0, \\
& \lim _{n \rightarrow \infty} \Delta^{2 j-2} z_{n}=\infty .
\end{aligned}
$$

In view of (3.51) and the monotonicity of $\left\{\Delta^{i} z_{n}\right\}$, we see that

$$
\lim _{n \rightarrow \infty} \Delta^{i} z_{n}=0, \quad i=2 j, 2 j+1, \ldots, m-1 .
$$

Now summing (2.3) $m-2 j+1$ times from $N_{0}+l$ to $n$ and invoking (3.53), we have

$$
\Delta^{2 j-1} z_{n+1}=\Delta^{2 j-1} z_{N_{0}+l}-\sum_{i_{2}=N_{0}+l}^{n} \sum_{i_{1}=i_{2}}^{\infty} \frac{\left(i_{1}-i_{2}+m-2 j-1\right)^{(m-2 j-1)}}{(m-2 j-1) !} f\left(i_{1}, x_{i_{1}-l}\right) .
$$

Noticing (3.50) and (3.51), we see that (3.44) holds.

By taking limits on both sides of (3.54) as $n \rightarrow \infty$ and then replacing $N_{0}+l$ by $n$, we see from (3.51) that

$$
\Delta^{2 j-1} z_{n}=\sum_{i_{1}=n}^{\infty} \frac{\left(i_{1}-n+m-2 j\right)^{(m-2 j)}}{(m-2 j) !} f\left(i_{1}, x_{i_{1}-l}\right), \quad n \geq N_{0} .
$$

Summing (3.55) from $N_{0}+l$ to $n$, we have

$$
\Delta^{2 j-2} z_{n+1}-\Delta^{2 j-2} z_{N_{0}+l}=\sum_{i_{2}=N_{0}+l}^{n} \sum_{i_{1}=i_{2}}^{\infty} \frac{\left(i_{1}-n+m-2 j\right)^{(m-2 j)}}{(m-2 j) !} f\left(i_{1}, x_{i_{1}-l}\right) .
$$

Invoking (3.49) and (3.52), we see that (3.45) holds.

Conversely, we demonstrate the sufficiency. Suppose that $-1<c_{0}<0$. Set $L_{n}=n^{(2 j-2)}$ and $R_{n}=n^{(2 j-1)}$. Take $c_{1}$ so that $-c_{0}<c_{1}<\left(1-4 c_{0}\right) / 5<1$. Similar to the proof of Theorem 3.1 , there exists an integer $N>k+l$ such that when $n \geq N$, we have

$$
\begin{gathered}
2 L_{n} \leq R_{n}, \quad-c_{n} \leq c_{1}, \quad \frac{|c| R_{n}}{R_{n-k-l}} \leq c_{1}, \\
\frac{L_{n-k}}{L_{n}} \geq \frac{1-5 c_{1}}{4 c_{n}}, \quad \sum_{i_{m-2 j+1}=N}^{\infty} \sum_{i_{m-2 j}=i_{m-2 j+1}}^{\infty} \cdots \sum_{i_{1}=i_{2}}^{\infty} f\left(i_{1},\left(i_{1}-l\right)^{(2 j-1)}\right)<\frac{1-c_{1}}{8} .
\end{gathered}
$$

Take $N_{0}=N-k-l, r_{n}=R_{n}^{2}$ and define the Banach space $l_{N_{0}}^{\infty}$ as in (1.4). Let

$$
\Omega=\left\{x \in l_{N_{0}}^{\infty}: L_{n} \leq x_{n} \leq R_{n}\right\}
$$


Define two operators on $\Omega$ as follows:

$$
\begin{aligned}
& (U x)_{n}= \begin{cases}\frac{1}{2} L_{n} & N_{0} \leq n<N \\
-\frac{3}{2} c_{1} L_{n}-c_{n} x_{n-k} & n \geq N,\end{cases} \\
& (S x)_{n}= \begin{cases}\frac{1}{2} L_{n} & N_{0} \leq n<N \\
\frac{3}{2} L_{n}+F(n) & n \geq N,\end{cases}
\end{aligned}
$$

where

$$
F(n)=\sum_{i_{m}=N}^{n-1} \cdots \sum_{i_{m-2 j+3}=N}^{i_{m-2 j+4}-1} \sum_{i_{m-2 j+2}=N}^{i_{m-2 j+3}-1} \sum_{i_{m-2 j+1}=i_{m-2 j+2}}^{\infty} \sum_{i_{m-2 j}=i_{m-2 j+1}}^{\infty} \cdots \sum_{i_{1}=i_{2}}^{\infty} f\left(i_{1}, x_{i_{1}-l}\right) .
$$

Analogous to the discussions in Theorem 3.1, there exists $x=\left\{x_{n}\right\} \in \Omega$ such that

$$
x_{n}=\frac{3}{2}\left(1-c_{1}\right) L_{n}-c_{n} x_{n-k}+F(n), \quad n \geq N
$$

From (3.61) and (3.46), we see that

$$
\lim _{n \rightarrow \infty} \frac{z_{n}}{n^{(2 j-1)}}=0
$$

By Lemma 1.2, we have

$$
\lim _{n \rightarrow \infty} \frac{x_{n}}{n^{(2 j-1)}}=0
$$

Note that (3.47) implies

$$
\lim _{n \rightarrow \infty} \Delta^{2 j-2} F(n)=\infty
$$

Hence, in view of (3.61) and Lemma 1.2, we see that

$$
\lim _{n \rightarrow \infty} \frac{x_{n}}{n^{(2 j-2)}}=\infty
$$

This means (1.1) has an eventually positive solution in $A_{2 j-1}(\infty, 0)$ when $-1<c_{0}<0$.

When $c_{0}=0$, we take $c_{1}$ so that $0<c_{1} \leq 1 / 3$ and the rest of proof is the same as the above and is thus skipped. The proof is complete.

A result similar to Theorem 3.5 is the following. 
16 A classification scheme for neutral difference equation

Theorem 3.6. Suppose $m$ is odd. If (1.1) has a solution in $A_{2 j}(\infty, 0)$ for some $j \in\{1,2, \ldots$, $(m-1) / 2\}$, then

$$
\begin{gathered}
\sum_{i=0}^{\infty} \frac{(i+m-2 j-1)^{(m-2 j-1)}}{(m-2 j-1) !} f\left(i,(i-l)^{(2 j-1)}\right)<\infty, \\
\sum_{i=0}^{\infty} \frac{(i+m-2 j)^{(m-2 j)}}{(m-2 j) !} f\left(i,(i-l)^{(2 j)}\right)=\infty .
\end{gathered}
$$

Conversely, if

$$
\begin{gathered}
\sum_{i=0}^{\infty} \frac{(i+m-2 j-1)^{(m-2 j-1)}}{(m-2 j-1) !} f\left(i,(i-l)^{(2 j)}\right)<\infty, \\
\sum_{i=0}^{\infty} \frac{(i+m-2 j)^{(m-2 j)}}{(m-2 j) !} f\left(i,(i-l)^{(2 j-1)}\right)=\infty,
\end{gathered}
$$

then (1.1) has a solution in $A_{2 j}(\infty, 0)$.

Theorem 3.7. Suppose $m$ is even and $c_{0}<0$. If there exist constants $\alpha>0, c_{1}$ with $0<c_{1}<$ $-c_{0}$ and integer $M>k+l$ such that

$$
c_{1} e^{\alpha k}>1
$$

as well as

$$
\sum_{i=n}^{\infty} \frac{(i-n+m-1)^{(m-1)}}{(m-1) !} f\left(i, \frac{1}{i-l}\right) \leq\left(c_{1} e^{\alpha k}-1\right) e^{-\alpha n}, \quad n \geq M,
$$

then (1.1) has a solution in $A_{0}(0)$.

Proof. First note that there exists integer $N>M$ such that

$$
\begin{gathered}
e^{-\alpha n}<\frac{1}{n}, \quad n \geq N-k-l, \quad-c_{n} \geq c_{1}, \quad n \geq N, \\
\frac{-c_{n}}{n-k} \leq \frac{1}{n}, \quad n \geq N, \\
\sum_{i=n}^{\infty} \frac{(i-n+m-1)^{(m-1)}}{(m-1) !} f\left(i, \frac{1}{i-l}\right) \leq\left(c_{1} e^{\alpha k}-1\right) e^{-\alpha n}, \quad n \geq N .
\end{gathered}
$$

Take $N_{0}=N-k-l, r_{n}=1$ and define the Banach space $l_{N_{0}}^{\infty}$ as in (1.4). Let

$$
\Omega=\left\{x \in l_{N_{0}}^{\infty}: e^{-\alpha n} \leq x_{n} \leq \frac{1}{n}\right\} .
$$

Define two operators on $\Omega$ as follows:

$$
\begin{gathered}
(U x)_{n}=0 \quad \text { for } n \geq N_{0}, \\
(S x)_{n}= \begin{cases}\frac{1}{n} & N_{0} \leq n<N \\
-c_{n} x_{n-k}-F(n) & n \geq N,\end{cases}
\end{gathered}
$$


where

$$
F(n)=\sum_{i_{m}=n}^{\infty} \sum_{i_{m-1}=i_{m}}^{\infty} \cdots \sum_{i_{2}=i_{3}}^{\infty} \sum_{i_{1}=i_{2}}^{\infty} f\left(i_{1}, x_{i_{1}-l}\right) .
$$

By arguments similar to those in the proof of Theorem 3.1, we may prove that there exists $x=\left\{x_{n}\right\} \in \Omega$ such that

$$
x_{n}=-c_{n} x_{n-k}+F(n), n \geq N .
$$

In view of the definition of $\Omega$, we see that $x$ is a solution of (1.1) in $A_{0}(0)$. The proof is complete.

A variant of Theorem 3.7 is the following and its proof is omitted.

Theorem 3.8. Suppose $m$ is odd and $c_{0}<0$. If there exist constants $\alpha>0, c_{1}, c_{2}$ with $0<$ $c_{1}<-c_{0}<c_{2}<1$ and integer $M>l+k$ such that

$$
c_{1} e^{\alpha k}>1
$$

as well as

$$
\sum_{i=n}^{\infty} \frac{(i-n+m-1)^{(m-1)}}{(m-1) !} f\left(i, \frac{1}{i-l}\right) \leq \frac{1}{n}-\frac{c_{2}}{n-k}, \quad n \geq M,
$$

then (1.1) has a solution in $A_{0}(0)$.

Theorem 3.9. Suppose that $m$ is odd. If (1.1) has a solution in $A_{0}(a)$ for some $a>0$, then there exists some $K>0$ such that

$$
\sum_{i=0}^{\infty} \frac{(i+m-1)^{(m-1)}}{(m-1) !} f(i, K)<\infty .
$$

The converse also holds.

The proof is similar to that of Theorem 3.1 and is skipped.

Example 3.10. Consider the equation

$$
\Delta^{2}\left(x_{n}-\frac{3}{4} x_{n-1}\right)+\frac{1}{16} x_{n-1}=0,
$$

here $f(n, x)=(1 / 16) x$.

It is clear that

$$
\sum_{i=0}^{\infty} f(i, K(i-1))=\infty, \quad \sum_{i=0}^{\infty}(i+1) f(i, K)=\infty
$$

for any $K>0$ and

$$
\sum_{i=0}^{\infty}(i+1) f(i, i-1)=\infty
$$


Hence by Theorems 3.1 and 3.3, an eventually positive solution of (3.78) cannot be in $A_{1}(\infty, a)$ nor in $A_{1}(a, 0)$. In addition, by Theorem 3.5 , an eventually positive solution of (3.78) cannot be in $A_{1}(\infty, 0)$. However, by Theorem 2.1, (3.78) has a solution in $A_{0}(0)$ if it has some eventually positive solution. Indeed, $\left\{x_{n}\right\}=\left\{1 / 2^{n}\right\}$ satisfies (3.78) and $\lim _{n \rightarrow \infty} x_{n}=0$.

Consider another equation

$$
\Delta^{3}\left(x_{n}-\frac{1}{5} x_{n-2}\right)+\frac{1}{80} x_{n-1}=0
$$

here $f(n, x)=(1 / 80) x$. Then, it is easy to see that

$$
\sum_{i=0}^{\infty} f\left(i, K(i-1)^{(2)}\right)=\infty, \quad \sum_{i=0}^{\infty}(i+1) f(i, K(i-1))=\infty,
$$

as well as

$$
\sum_{i=0}^{\infty} \frac{(i+2)^{(2)}}{2} f(i, K)=\infty
$$

for any $K>0$, and

$$
\sum_{i=0}^{\infty} f(i, i-1)=\infty
$$

By the similar reasons to the above, (3.81) has a solution in $A_{0}(0)$ if it has some eventually positive solution. Indeed, $\left\{x_{n}\right\}=\left\{1 / 2^{n}\right\}$ is such a solution of (3.81).

\section{References}

[1] R. P. Agarwal, Difference Equations and Inequalities. Theory, Methods, and Applications, Monographs and Textbooks in Pure and Applied Mathematics, vol. 155, Marcel Dekker, New York, 1992.

[2] Y. S. Chen, Asymptotic behavior of nonoscillatory solutions of higher order neutral delay differential equations, Annals of Differential Equations 9 (1993), no. 3, 270-286.

[3] S. S. Cheng, Partial Difference Equations, Taylor \& Francis, London, 2003.

[4] S. S. Cheng, G. Zhang, and W.-T. Li, On a higher order neutral difference equation, Mathematical Analysis and Applications (Th. M. Rassias, ed.), Hadronic Press, Florida, 2000, pp. 37-64.

[5] X. Z. He, Oscillatory and asymptotic behaviour of second order nonlinear difference equations, Journal of Mathematical Analysis and Applications 175 (1993), no. 2, 482-498.

[6] B. S. Lalli, Oscillation theorems for neutral difference equations. Advances in difference equations, Computers \& Mathematics with Applications 28 (1994), no. 1-3, 191-202.

[7] W.-T. Li, S. S. Cheng, and G. Zhang, A classification scheme for nonoscillatory solutions of a higher order neutral nonlinear difference equation, Journal of the Australian Mathematical Society. Series A 67 (1999), no. 1, 122-142.

[8] X. Tang and J. Yan, Oscillation and nonoscillation of an odd-order nonlinear neutral difference equation, Functional Differential Equations 7 (2000), no. 1-2, 157-166 (2001).

[9] G. Zhang and S. S. Cheng, Oscillation criteria for a neutral difference equation with delay, Applied Mathematics Letters 8 (1995), no. 3, 13-17. 
[10] B. Zhang and Y. J. Sun, Classification of nonoscillatory solutions of a higher order neutral difference equation, Journal of Difference Equations and Applications 8 (2002), no. 11, 937-955.

[11] B. G. Zhang and B. Yang, Oscillation in higher-order nonlinear difference equations, Chinese Annals of Mathematics. Series A 20 (1999), no. 1, 71-80 (Chinese).

Zhi-qiang Zhu: Department of Computer Science, Guangdong Polytechnic Normal University, Guangzhou, Guangdong 510665, China

Gen-qiang Wang: Department of Computer Science, Guangdong Polytechnic Normal University, Guangzhou, Guangdong 510665, China

Sui Sun Cheng: Department of Mathematics, Tsing Hua University, Hsinchu, Taiwan 30043, China E-mail address: sscheng@math.nthu.edu.tw 